\title{
PULSO DE OXIGÊNIO: PREDIÇÃO DA PRODUÇÃO DE CALOR EM BOVINOS E RELAÇÕES COM CONSUMO ALIMENTAR RESIDUAL
}

\author{
OXYGEN PULSE, PREDICTION OF HEAT PRODUCTION IN BOVINES AND \\ RESIDUAL FEED INTAKE
}

\author{
Chaves, S.A. ${ }^{1 *}$; Feltrin, G.B. ${ }^{1}$; Nascimento, M.L. ${ }^{1}$ e Lanna, D.P.D. ${ }^{2}$ \\ ${ }^{1}$ Programa de Pós Graduação em Ciência Animal e Pastagens. Universidade de São Paulo. Campus ESALQ. \\ Piracicaba-SP. Brasil. \\ 2Departamento de Zootecnia.Universidade de SãoPaulo.Campus ESALQ. *amaliaschaves@yahoo.com.br
}

\section{PalaVRas chaVe adicionais}

Bioenergética. Consumo de oxigênio. Eficiência alimentar. Frequência cardíaca.

\section{RESUMO}

O interesse quanto ao uso de índices de eficiência alimentar na seleção de bovinos de corte é crescente, tanto pela redução no custo de produção quanto no impacto ambiental, questão esta de grande importância, visto que o foco atualmente prioriza a sustentabilidade dos recursos naturais. O consumo alimentar residual (CAR) obtido pela diferença entre o consumo observado e predito, vem sendo estudado como um índice de eficiência alimentar que além de evitar o aumento do tamanho adulto dos animais selecionado, por ser ajustado para peso metabólico, possibilita a identificação de indivíduos com menor consumo de matéria seca ao mesmo peso e taxa de ganho. Características de desempenho e suas correlações com a eficiência alimentar são bem documentadas na literatura, entretanto as bases biológicas para variação no CAR são parcialmente desconhecidas. Estima-se que $9 \%$ das diferenças em CAR são explicadas pelo incremento calórico, $14 \%$ por processo de digestão, $5 \%$ pela composição corporal e $5 \%$ por diferenças em atividade, Desta forma, $67 \%$ da variação do CAR permanece ainda desconhecida, e pode estar relacionada com a energia requerida pelos processos biológicos, como bombeamento de prótons na mitocôndria, turnover proteico e bombeamento de íons. Portanto, o uso da energia via processos biológicos parece ter um potencial de contribuição com substancial proporção na variação individual da eficiência alimentar. Embora

\section{AdDitiOnAL KEYWORDS}

Bioenergetics. Feed efficiency. Heart rate. Oxygen consumption.

a produção de calor de um animal possa ser medida de forma acurada por meio de câmara calorimétrica ou uso de água duplamente marcada, estes métodos, além de serem praticados em condições artificiais, são extremamente caros e requerem considerável experiência e infraestrutura, o que pode tornar impraticável a seleção de animais com menor exigência. Neste contexto, muitos pesquisadores exploram a possibilidade de predizer a produção de calor a partir da frequência cardíaca, uma vez que a maior parte do $\mathrm{O}_{2}$ utilizado por animais homeotermos é transportada aos tecidos pela ação do coração, órgão este cuja atividade representa cerca de 10 \% da produção total de calor. O uso da frequência cardíaca, sem a calibração para o volume de $\mathrm{O}_{2}$ consumido por batimento cardíaco, pode apresentar estimativas de baixa acurácia, no entanto, a produção de calor estimada por esta calibração em humanos e animais é altamente correlacionada com a mensuração direta na câmara calorimétrica. Diante disto, o objetivo na presente revisão é apresentar a metodologia de estimativa da produção de calor por meio da frequência cardíaca calibrada para consumo de oxigênio, e sua relação com o CAR em bovinos de corte.

\section{SUMMARY}

The interest on the use of feed efficiency 
indexes in beef cattle selection is increasing, by reduction on production costs and on environmental impact, what is an important issue, since currently the focus is on the natural resources sustainability. Residual feed intake (RFI) obtained by the difference between observed and predicted dry matter intake, has been studied as a feed efficiency index that besides avoiding increase on body weight of the selected animals, for being adjusted for metabolic body weight, allows the identification of the individuals with lower dry matter intake with the same body weight and average daily gain. Performance characteristics and their correlations with feed efficiency are well reported by the literature, however the biological basis for RFI variation are partially unknown. It is estimated that $9 \%$ of the difference on RFI are explained by heat increment, $14 \%$ for differences in digestion; $5 \%$ for differences in body composition; and $5 \%$ for differences in activity. Then, $67 \%$ of the RFI variance remains unknown and may be related to the energy required for biological processes such as pumping of protons into the mitochondria, protein turnover and ion pumping. Therefore, the energy use through biological processes seems to have a potential to contribute substantially on individual variation of feed efficiency. Although the heat production of an animal can be accurately measured by calorimetric chamber or using doubly labeled water, these methods, besides being practiced in artificial conditions are extremely expensive and require considerable experience and infrastructure, which may become impractical selecting animals with lower requirement of maintenance. In this context, many researchers have been studying the possibility of predicting heat production from heart rate, since most of the $\mathrm{O}_{2}$ used by warmblooded animals are transported to the tissues by heart pumping, an organ whose activity is about $10 \%$ of the total heat production. The use of heart rate without calibration for the $\mathrm{O}_{2}$ volume consumed per heart beat can provide low accuracy estimates, however, the heat production estimated by that calibration in humans and animals is highly correlated with the direct measurement in calorimetry chamber. Hence, the aim of this review is presenting the methodology of estimation of heat production from heart rate calibrated to oxygen consumption and its relationship with RFI in beef cattle.

\section{INTRODUÇÃO}

Existe um crescente interesse quanto ao uso de índices de eficiência alimentar em bovinos em crescimento, devido ao fato da conversão de alimento em produto de origem animal durante esta fase ter uma larga influência no custo de produção (Retallick et al., 2013; Herd et al., 2003). Uma vez que a rentabilidade é dependente dos inputs e dos outputs do sistema, e o fornecimento de alimentos é o input de maior custo na maioria dos empreendimentos pecuários (Arthur et $a l ., 2004)$. Com isto, a identificação de animais mais eficientes na fase de crescimento é de grande importância para melhorar economicamente e ambientalmente a indústria de bovinos de corte (Herd et al., 2003; Arthur et al., 2004; Lancaster et al., 2009). Contudo, a principal desvantagem da grande maioria dos índices de eficiência alimentar é a necessidade de medir consumo individual dos animais, em que o custo de mensuração da eficiência em bovinos individualmente em confinamentos comerciais ou em pesquisas científicas é relativamente alto, limitando o uso da inclusão da eficiência alimentar em programas de melhoramento de bovinos (Herd et al., 2003; Arthur et al., 2004).

Uma maneira de reverter esta situação seria a identificação de animais com menor exigência de mantença. O custo energético de mantença, perdido na forma de calor, pode representar $70-75 \%$ do consumo total de energia (Ferrell e Jenkins, 1984; NRC, 1984), e as diferenças nas exigências de mantença, que podem variar de 10 a $12 \%$ em bovinos de corte, são componentes chaves que definem as variações na eficiência entre animais (Hotovy et al., 1991; Swanson e Miller, 2008). Estas variações, juntamente com as estimativas de herdabilidade de moderada a alta $\left(\mathrm{h}^{2}=0,22\right.$ a 0,71$)$, sugerem amplo espaço para melhorias através da seleção também de animais com menor exigência de mantença, ou seja, animais que perdem menos energia na forma de calor 


\section{PULSO DE OXIGÊNIO, PRODUÇÃO DE CALOR E CONSUMO ALIMENTAR RESIDUAL}

(Carstens et al., 1989; Bishop et al., 1991). Embora a produção de calor de um animal possa ser medida de forma acurada por meio de câmara calorimétrica (Rodriguez et $a l ., 2007)$, ou uso de água duplamente marcada (Fancy et al., 1986), estes métodos, além de serem praticados em condições artificiais, são extremamente caros e requerem considerável experiência e infraestrutura, o que pode tornar impraticável a seleção de animais com menor exigência. Neste contexto, ao longo das últimas décadas esforços significantes têm sido somados por pesquisadores na tentativa de desenvolver métodos para estimar a produção de calor de animais em seus ambientes naturais. Muitos pesquisadores exploraram a possibilidade de predizer a produção de calor a partir da frequência cardíaca (Webster, 1967; Bradfield et al., 1971; Payne et al., 1971; Warnold e Lenner, 1977), uma vez que a maior parte do O2 utilizado por animais homeotermos é transportada aos tecidos pela ação do coração, órgão este cuja atividade representa cerca de $10 \%$ da produção total de calor. O uso da frequência cardíaca, sem a calibração para o volume de $\mathrm{O}_{2}$ consumido por batimento cardíaco, pode apresentar estimativas de baixa acurácia, no entanto, a produção de calor estimada por esta calibração em humanos é altamente correlacionada com a mensuração direta na câmara calorimétrica ( $\mathrm{r}=0,943)$ (Ceesay et al., 1989). Como a frequência cardíaca varia de acordo com as características de cada indivíduo a calibração deve ser feita individualmente (McCrory et al., 1997; Brosh et al., 1998a). Dessa forma, a frequência cardíaca calibrada para consumo de oxigênio poderia ser utilizada na identificação de animais com menor produção de calor, e consequentemente mais eficientes quanto à utilização do alimento (Brosh, 2007).

Diante disto, o objetivo no presente estudo é apresentar uma revisão sobre a metodologia que estima a produção de calor por meio da frequência cardíaca calibrada para consumo de oxigênio e sua relação com eficiência alimentar em bovinos.

\section{CONSUMO ALIMENTAR RESIDUAL}

Existe uma variedade de índices para descrever a eficiência alimentar, os quais, cada um à sua maneira, reflete diferenças biológicas e aspectos matemáticos, bem como suas interações com o ambiente (Arthur et al., 2004), sendo que desde 1960, mais de uma dúzia de índices e cálculos alternativos pra medir eficiência alimentar tem sido proposta na literatura científica (Archer et al., 1999). Contudo, a principal desvantagem da grande maioria dos índices de eficiência alimentar é a necessidade de medir consumo individual dos animais.

Precisão de registro de dados e correto período de tempo para mensuração para todos os índices também é importante (Robinson e Oddy, 2004), como é o caso de todas as medidas de desempenho. O período de tempo de mensuração necessária para obter estimativas acuradas de eficiência alimentar foi discutido em alguns trabalhos (Archer et al., 1997; Archer e Bergh, 2000; Wang et al., 2006; Castilhos et al., 2011). Archer et al. (1997) constataram que seriam necessários no mínimo 35 dias de avaliação para animais taurinos. Para a raça Nelore considerou-se que 28 dias de avaliação são suficientes (Castilhos et al., 2011). No entanto, para o ganho de peso, deve-se ter um período mínimo de avaliação de 70 a 84 dias em função da maior variação desta característica no tempo (devido às diferenças de conteúdo gastrointestinal entre pesagens) quando comparado ao consumo. Por esta razão o CAR deve ser determinado por um período mínimo de 70 dias (Archer; Bergh, 2000; Castilhos et al., 2011).

O CAR tem crescido em popularidade como índice para mensurar eficiência alimentar, e tem sido proposto como um método que pode ser utilizado na seleção genética de animais mais eficientes (Archer et al., 1997; Arthur et al., 2001a; Berry, 2008; 
Berry e Crowley, 2013), desde que foi primeiramente sugerido para o uso em bovinos por Koch et al. (1963). Esse índice é calculado como sendo a diferença entre o consumo individual observado e aquele predito por regressão múltipla do CMS observado em função do GPD e peso vivo metabólico (Koch et al., 1963). Desta forma, esta medida tem o importante benefício de ser ajustada para peso e ganho do animal, o que não acarretaria em aumento do peso adulto, comparado, por exemplo, com conversão alimentar.

O CAR pode ser dividido em dois parâmetros: 1) consumo previsto para o nível de mantença e produção (ganho de peso e peso), e 2) uma parcela residual, que poderia ser utilizada para identificar animais que tenham desvio do nível de consumo esperado (Koch et al., 1963). Portanto, com esse índice é possível identificar animais que apresentem diferença entre a exigência nutricional estimada e observada e classificá-los como animais eficientes aqueles que se mantém e crescem com consumo inferior ao estimado, e animais ineficientes, aqueles que se mantém e crescem consumindo acima do valor estimado (Del Claro, 2011). Com isto, animais mais eficientes apresentam CAR negativo e consomem menos alimento que seus contemporâneos, para expressarem o mesmo ganho de peso para a mesma faixa de peso vivo.

Resultados de vários estudos indicam que este índice é moderadamente herdável e geneticamente independente das características de crescimento e, ainda, que existe uma considerável variação genética para CAR e características produtivas, o que sugere que este índice pode ser explorado na identificação e seleção de animais geneticamente superiores em relação ao uso dos alimentos para produção de carne (Herd e Bishop, 2000; Arthur et al., 2001a; Schenkel et al., 2004; Nkrumah et al., 2007; Ahola et al., 2011; Berry e Crowley, 2013). Estima-se que a herdabilidade para CAR está entre 0,13 e 0,82 (Jensen et al., 1992; Arthur et al., 2001ab; Robinson e Oddy, 2004; Nkrumah et al., 2007; Lancaster et al., 2009; Crowley et al., 2010; Berry e Crowley, 2013). Arthur et al. (2001ab) analisando consumo individual em machos e fêmeas da raça Angus e Charolês relataram estimativa de herdabilidade para CAR de 0,39 $\pm 0,03$, em ambos os estudos. Robinson e Oddy (2004), com grande número de machos castrados e fêmeas provenientes de raças adaptadas aos trópicos e de raças temperadas, relataram estimativa de herdabilidade inferior $(0,18)$ para CAR, provavelmente pelo fato dos animais serem mais velhos e mais pesados que nos estudos anteriores. Enquanto, Nkrumah et al. (2007) relataram estimativa de herdabilidade para CAR de 0,42 $\pm 0,15$ em machos castrados provenientes de cruzamentos entre Bos taurus. Com isto, estes dados confirmam a existência de considerável variabilidade genética da característica CAR em vários genótipos e ambientes.

\section{PRODUÇÃODECALOR}

Características de desempenho e suas correlações com CAR são bem documentadas na literatura, entretanto as bases biológicas para variação na eficiência alimentar dos animais são pouco conhecidas. Estimase que 14, 9,5 e $5 \%$ da variação do CAR pode ser relativa ao processo de digestão, incremento calórico, composição corporal e atividades físicas, respectivamente; desta forma. Então, 67 \% da variação do CAR permanece ainda inexplicável, e que pode estar relacionada com a energia requerida pelos processos biológicos, como bombeamento de prótons na mitocôndria, turnover proteico e bombeamento de íons (Herd et al., 2004). Portanto, o uso da energia via processos biológicos parece ter um potencial de contribuição com substancial proporção na variação individual da eficiência alimentar (Hill e Herd, 2001).

Tem sido aceito na literatura que os 


\section{PULSO DE OXIGÊNIO, PRODUÇÃO DE CALOR E CONSUMO ALIMENTAR RESIDUAL}

principais fatores que afetam a partição de energia nos ruminantes são: nível de consumo alimentar, condições ambientais, gasto energético ou produção de calor (PC), nível de produção de leite ou ganho em tecido corporal e variabilidade individual entre animais com respeito à eficiência de utilização de energia para mantença e produção (Brosh, 2007). Ao calcular o equilíbrio energético do animal, a produção de calor representa um componente substancial do balanço de energia dos ruminantes (Castro Bulle et al., 2007). Em estudos de metabolismo energético a produção de calor dos animais é estimada como produção de calor total, que inclui o calor utilizado para mantença, adicionado ao calor despendido na forma de incremento calórico (Rodriguez et al., 2007). A exigência de mantença pode ser definida como a quantidade de energia necessária para animais com zero de ganho de peso (Ferrell e Jenkins, 1984). Mais especificamente, essas necessidades representam a quantidade de energia necessária para manter os processos do metabolismo basal (síntese e degradação proteica, transporte de íons, sinalização celular, etc), função de órgãos vitais, movimentos voluntários e termorregulação (Thompson et al., 1983).

O custo energético de mantença, perdido na forma de calor, que pode representar 70-75\% do consumo total de energia (Ferrell e Jenkins, 1984; NRC, 1984), e as diferenças nas exigências de mantença, que podem variar de 10 a $12 \%$ em bovinos de corte, são componentes chaves que definem as variações na eficiência entre animais (Hotovy et al., 1991; Swanson e Miller, 2008). Estas variações, juntamente com as estimativas de herdabilidade de moderada a alta $\left(h^{2}=0,22\right.$ a 0,71$)$, sugerem amplo espaço para melhorias através de seleção também de animais com menor exigência de mantença, ou seja, animais que perdem menos energia na forma de calor (Carstens et al., 1989; Bishop et al., 1991).

A produção de calor pode ser mensurada por calorimetria direta, pela medição direta do calor liberado pelo animal, ou indireta em câmara calorimétrica (Rodriguez et al., 2007), pelo uso de água duplamente marcada (Fancy et al., 1986). A calorimetria direta é simples na teoria, mas difícil na prática. Projetos calorimétricos para estimar produção de calor em animais são baseados no mesmo princípio da bomba calorimétrica, em que o calor liberado é utilizado para aumentar a temperatura que circula no meio. Dentro da câmara, os gases são analisados para determinar, indiretamente, o metabolismo dos alimentos e, diretamente, a dissipação de calor (evaporação, radiação, condução e convecção) pelo aumento da temperatura da água circundante na câmara, sendo que um kcal de energia equivale ao aumento da temperatura de um grama de água de 14,5 para $15,5^{\circ} \mathrm{C}$ em condições normais de temperatura e pressão. Este não é um método que pode ser utilizado a campo, além de ser complexo e de custo elevado (Kleiber, 1961).

\section{CALORIMETRIAINDIRETA}

A calorimetria indireta é uma técnica que quantifica o calor gerado pelas reações químicas, ou seja, é uma medida metabólica da oxidação de compostos orgânicos. Ela foi desenvolvida na virada do século XIX para o XX, como uma aplicação da termodinâmica para vida animal (Van Soest, 1982). É baseada no conhecimento da combustão do substrato energético ingerido e necessário pelo organismo. Os diferentes tipos de nutrientes têm quantidades específicas de $\mathrm{O}_{2}$ consumido e $\mathrm{CO}_{2}$ produzido, portanto, a produção de calor é obtida através do quociente respiratório (QR), o qual é determinado pelo balanço conjunto de oxigênio e carbono, ou das perdas e ganhos de tecidos pelos animais (Kleiber, 1961; Van Soest, 1982).

Existem diversas técnicas de se medir as trocas gasosas na respiração, sendo que todas elas levam em consideração o consumo de oxigênio $\left(\mathrm{VO}_{2}\right)$ e a produção de $\mathrm{CO}_{2}$ por unidade de tempo. Os aparelhos de 
calorimetria que medem $\mathrm{VO}_{2}$ e $\mathrm{CO}_{2}$ analisando o ar inspirado e expirado podem ser classificados em equipamentos de circuito fechado ou circuito aberto, e têm sido utilizados desde 1900 em humanos e animais (Kleiber, 1961). Nos equipamentos de circuito fechado, $\mathrm{O} \mathrm{VO}_{2}$ e $\mathrm{CO}_{2}$ são medidos por alterações no volume dentro de um reservatório fechado contendo oxigênio. Os aparelhos consistem de um espirômetro de selo d'água contendo oxigênio a 100 \%, um filtro com cal-sodada para absorver o gás carbônico e um circuito respiratório com válvula inspiratória e válvula expiratória. O indivíduo respira continuamente o gás contido no espirômetro por meio do sistema de válvulas direcionais. A redução no volume do gás contido no espirômetro, no período do exame, permite determinar o consumo do oxigênio. Embora ainda utilizados para estudos de calorimetria de pequenos animais, poucos equipamentos para uso em bovinos empregam esse princípio (Ferrannini, 1988).

$\mathrm{Na}$ calorimetria indireta de circuito aberto, ambos os extremos do sistema se comunicam com o ambiente. $\mathrm{O}$ ar inspirado é mantido separado do ar expirado por meio de um sistema de válvulas unidirecionais. Nos estudos de fisiologia e, ocasionalmente, em estudos clínicos, o ar expirado é coletado em bolsa de Douglas ou espirômetro de Tissot e, depois, analisado por titulação por métodos convencionais ou analisadores eletrônicos (Kleiber, 1961). Os tradicionais métodos que utilizam a calorimetria indireta para mensurar o gasto energético são as câmaras calorimétricas de circuito aberto onde os animais são alojados individualmente. Embora seja uma medida acurada, essas câmaras não representam o real ambiente do animal e restringem as atividades dos animais, o que pode afetar as exigências de energia e seu uso de acordo com NRC (1996).

Técnicas como água duplamente marcada e intubação traqueal são algumas formas para estimar a produção de calor por calorimetria indireta sem uso de aparelhos. A água duplamente marcada pode ser utilizada para determinar a produção de $\mathrm{CO}_{2}$, e mensurar a taxa metabólica em condiçõ̃es naturais dos animais, contudo tem custo elevado e o método contem erros de 8 a 11 \% (Nagy, 1989). Intubação traqueal para mensurar o consumo de oxigênio (Young e Webster, 1963) e infusão de [14C] bicarbonato (Yang e Cobertt, 1972) tem restrita aplicação e custo elevado para o uso em grandes animais. Além disto, estes métodos interferem no comportamento normal dos animais.

Embora a produção de calor de um animal possa ser medida de forma acurada por meio de câmara calorimétrica, estes métodos, além de serem praticados em condições artificiais, são extremamente caros e requerem considerável experiência e infraestrutura, o que pode tornar impraticável a seleção de animais com menor exigência.

\section{Metodologiado pulso de $\mathrm{O}_{2}\left(\mathrm{O}_{2} \mathrm{P}\right)$}

O uso de frequência cardíaca (FC) como indicador de gasto energético, comumente chamado de produção de calor (PC), em ovelhas e bovinos foi sugerido anteriormente (Webster, 1967; Yamamoto et al., 1979). Recentes desenvolvimentos em microeletrônica permitiram que a frequência cardíaca dos animais fosse monitorada em seu ambiente natural, incluindo animais sobre pastejo ou confinados (Brosh et al., 1998a; Aharoni et al.,1999). A correlação entre produção de calor e FC em humanos (Andrews, 1971) e animais (Webster, 1967), e a relativa simplicidade de mensuração da FC, fez com que continua mensuração de FC pudesse ser uma técnica em potencial para determinar a produção de calor em animais em seus ambientes naturais.

Porém, ao utilizar somente a FC como preditora da PC a mesma pode fornecer estimativas incorretas devido aos efeitos do nível de atividade, variações diárias e individuais dos animais, dietas e outras 
condições fisiológicas não consideradas (Yamamoto, 1989; Brosh, 2007).

Em mamíferos o oxigênio usado é transportado para os tecidos pelo coração, e este órgão representa $10 \%$ do consumo total de energia corporal. Portanto, ao ajustarmos individualmente a FC pelo consumo de oxigênio $\left(\mathrm{VO}_{2}\right)$ é possível acessar o volume de $\mathrm{O}_{2}$ bombeado por batimento, ou seja, o pulso de $\mathrm{O}_{2}$, e este por sua vez multiplicado pelo total de batimentos diários é capaz de produzir estimativas da produção de calor de maneira bastante acurada tanto em hu-
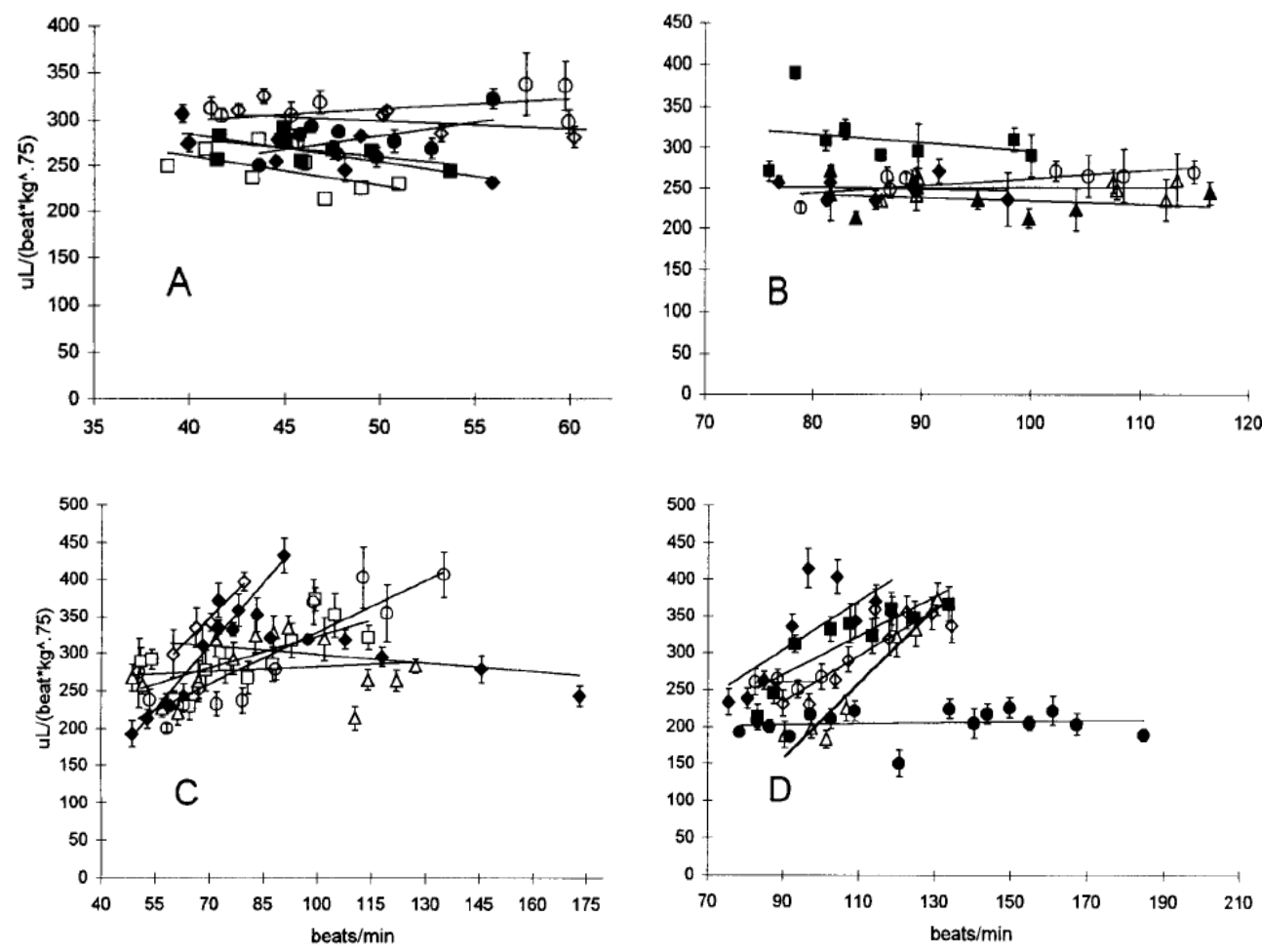

Figura 1. A e B) Pulso de oxigênio ( $\mu l$ / batimento*kg 0,75) e frequência cardíaca (batimentos/minuto) de 6 novilhas confinadas em descanso alimentadas com dietas de baixo e alto energia metabolizável, respectivamente. C e D) Novilhas em exercício utilizando uma esteira alimentadas com dieta de baixa e alta energia metabolizável, respectivamente. Adaptado de Brosh et al. (1998a). ((A and B) Pulse oxygen ( $\mu \mathrm{l} /$ beat $\left.^{*} \mathrm{~kg} 0.75\right)$ and heart rate (beats $/ \mathrm{min}$ ) of 6 heifers fed diets with low resting and high metabolizable energy, respectively. $C$ and $D$ ) heifers fed treadmill exercise using a diet with low and high metabolizable energy, respectively. Adapted from Brosh et al. (1998)). 
Aharoni et al. (2003) e Brosh et al. (1998a) sugeriram que para uma adequada estimativa do volume de $\mathrm{O}_{2}$ consumido por dia, e consequentemente da PC, o batimento cardíaco deve ser monitorado por cerca de quatro dias consecutivos e o consumo de oxigênio por batimento cardíaco $\left(\mathrm{O}_{2} \mathrm{P}\right)$ por um período de 15 a 20 minutos.

O que permite que essa metodologia seja utilizada em períodos curtos de avaliação é o fato de o $\mathrm{O}_{2} \mathrm{P}$ de cada indivíduo ser considerado constante, desde que o animal não esteja em condições de estresse (Aharoni et al., 2003). Se os animais estiverem estressados ao ponto de elevar a FC em mais de $20 \%$ daquela expressa em condições normais (Brosh, 2007) o $\mathrm{O}_{2} \mathrm{P}$ deixa de ter uma relação linear com a frequência cardíaca e passa a ter uma relação quadrática, o que produz estimativas superestimadas da produção de calor (figura 1). É importante, então, que as pessoas que lidam com os animais tanto no momento da mensuração do oxigênio, quanto no manejo diário, estejam cientes deste problema e minimizem ao máximo o estresse dos animais. Ainda, as mensurações de oxigênio devem ser realizadas somente depois que a FC normal do animal foi estabelecida, por isso é importante que a média diária da FC individual de cada animal seja mensurada e conhecida antes da avaliação do consumo de oxigênio (Aharoni et al., 2003; Brosh, 2007).

Além da condição de estresse dos animais a acurácia desta metodologia pode ser afetada por algumas variáveis, tais como, a variação do $\mathrm{O}_{2} \mathrm{P}$ ao longo do dia e sob diferentes condições ambientais, principalmente em termos de temperatura e o número de calibrações por animal. Alguns autores avaliaram as variações de $\mathrm{O}_{2} \mathrm{P}$ durante os períodos da manhã e a tarde e não observaram mudanças de $\mathrm{O}_{2} \mathrm{P}$ nos animais avaliados (Brosh et al., 1998b; Aharoni et al., 2003). Aharoni et al. (2003) avaliando bezerros e cordeiros observaram que mudanças no horário de mensuração do $\mathrm{VO}_{2}$ levaram a significativas flutuações de
FC, porém o $\mathrm{O}_{2} \mathrm{P}$ permaneceu constante. Além disto, nos animais submetidos a estresse térmico a FC foi afetada, e houve uma tendência de mudança no $\mathrm{VO}_{2}$, mas $\mathrm{O}_{2} \mathrm{P}$ não foi alterado.

$\mathrm{O}$ erro estimado quando o $\mathrm{O}_{2} \mathrm{P}$ é mensurado somente uma vez é de $10 \%$, enquanto, mensuração de $\mathrm{O}_{2} \mathrm{P}$ mais que uma vez no mesmo animal/dia pode diminuir o erro por um fator raiz quadrada de n, quando n representa o número de mensurações (Aharoni et al., 2003).

Vários trabalhos estudaram o uso do $\mathrm{O}_{2} \mathrm{P}$ para mensurar a produção de calor em ruminantes. Brosh et al. (1998b) avaliaram 6 novilhas Hereford em diferentes condições, a sombra ou expostas a radiação solar, alimentadas com dietas de alta e baixa energia, e as avaliações de consumo de oxigênio foram realizadas no período da manhã ou tarde. Eles observaram que a média diária da FC (52 \pm 4 bat/min) e a média do gasto energético diário $(380 \pm 9 \mathrm{Kj} / \mathrm{kg} 0,75)$ em animais alimentados com baixa densidade energética na dieta foi menor em relação aos animais alimentados com dieta de alta energia $(94 \pm 4$ bat $/ \mathrm{min}$ e $653 \pm 9 \mathrm{Kj} / \mathrm{kgPV} 0,75)$. Além disto, eles observaram que o $\mathrm{O}_{2} \mathrm{P}$ foi pouco afetado pelo nível de energia da dieta e não houve mudanças do $\mathrm{O}_{2} \mathrm{P}$ entre as mensurações feitas de manhã̃ ou à tarde. Eles recomendaram que sempre que o gasto energético é estimado pela $\mathrm{FC}$, o $\mathrm{O}_{2} \mathrm{P}$ de cada animal deve ser mensurado sob condições similares, e ainda concluíram que para aumentar a acurácia desta análise, a relação entre FC e PC deve ser estabelecida para cada animal individualmente.

Adicionalmente, Aharoni et al. (2003) realizaram três experimentos, um com bezerros machos com 2 meses de idade, outro com cordeiros com 5 meses e o terceiro com vacas de leite, para avaliar o $\mathrm{O}_{2} \mathrm{P}$ nos diferentes momentos do dia e debaixo de diferentes cargas de calor. O estresse calórico afetou a FC e tendeu a afetar o $\mathrm{VO}_{2}$, mas não alterou o $\mathrm{O}_{2} \mathrm{P}$ nos bezerros e cordeiros. Por outro lado, a $\mathrm{FC} \mathrm{e}_{2} \mathrm{P}$ foram fortemente 


\section{PULSO DE OXIGÊNIO, PRODUÇÃO DE CALOR E CONSUMO ALIMENTAR RESIDUAL}

relacionados com estresse calórico nas vacas de leite.

Com o objetivo de demonstrar o potencial da produção de calor para caracterizar eficiência da energia bruta e líquida em animais Holandeses ou animais cruzados Holandês x Montbeliarde, Aharoni et al. (2006), observaram que os animais cruzados apresentaram menor FC, contudo isso não refletiu em diferenças no $\mathrm{O}_{2} \mathrm{P}$ e na produção de calor dos animais. Porém encontram menor consumo de oxigênio por unidade de peso vivo metabólico nos animais cruzados em relação aos Holandeses puros.

Apesar de o gasto energético, também poder ser mensurado por outros métodos como o abate comparativo ou pela diferença entre consumo de energia metabolizável e energia retida (ER), a utilização é limitada devido à dificuldade de estimar a ER em animais em seus ambientes naturais por curto período de tempo. Com isto, a metodologia utilizando FC pode ser aplicada em rebanhos comerciais em maior escala, ao contrário de quando se utiliza normalmente medições individuais de consumo para estimar o consumo de energia metabolizável (Aharoni et al., 2006). Arieli et al. (2002) conduziu um experimento com cordeiros com o objetivo de validar o método de FC para estimar a PC em relação àquela obtida pelo método de abate comparativo. A produção de calor obtida pelo $\mathrm{O}_{2} \mathrm{P}$ foi 6,7 \% maior em relação ao abate comparativo. Contudo, quando se estima produção de calor por calorimetria e abate comparativo, os valores podem variar de 2 a $10 \%$ (Close, 1990).

Dados na literatura relacionando produção de calor e índices de eficiência alimentar são escassos, principalmente quando a produção de calor é mensurada pela metodologia de $\mathrm{O}_{2} \mathrm{P}$, entretanto Alguns trabalhos (Paddock, 2010; Hafla, et al., 2013; Chaves, et al., 2013, 2014) foram encontradas para ilustrar esta relação. Hafla et al., (2013) não estudou a relação do CAR com a produção de calor, mas observou como a classificação de CAR pode afetar algumas características de desempenho em 48 novilhas Bonsmara no meio da gestação, dentre elas a FC. O autor observou que a FC das fêmeas classificadas em baixo CAR foi $7 \%$ menor em relação às fêmeas classificas em alto CAR (66,1 vs 71,1 bat/min). Paddock (2010) estudou 16 novilhas Brangus selecionadas para alto e baixo CAR e observou maior FC nos animais de alto CAR (89,6 vs 97,7 bat/min) em detrimento dos animais de baixo CAR. Além disto, as novilhas com alto CAR consumiram mais oxigênio por batimento cardíaco (ml/bat) e como resultado, o gasto energético foi 17,4 $\%$ maior nos animais de alto CAR comparado aos animais de baixo CAR.

Apesar disto Chaves et al. $(2013,2014)$ não observaramefeito significativo do CAR para batimentos cardíacos e para produção de calor avaliada por esta metodologia em bovinos Nelore. Neste trabalho foram monitorados 39 animais quanto à frequência cardíaca, a qual foi calibrada para consumo de $\mathrm{O}_{2}$ em 18 deles. Entretanto os autores observaram que a FC diária durante a calibração de $\mathrm{O}_{2}$ foi menor quanto maior a eficiência de acordo com o CAR (80,7 vs 91,6 bat/min; $\mathrm{p}<0,05)$, enquanto no período de avaliação de 4 dias, apesar de numericamente menor nos animais eficientes, a FC não foi relacionado ao CAR. Os animais ineficientes apresentaram batimento cardíaco médio $3 \%$ maior no momento da calibração em relação ao batimento medido no período de avaliação, que pode ser devido à maior resposta ao stress nestes animais. $\mathrm{O}$ consumo de $\mathrm{O}_{2}$ tanto em L/dia quanto em $\mathrm{ml} / \mathrm{bat}$ não se mostrou relacionado ao CAR, consequentemente os valores de $\mathrm{VO}_{2}$ e $\mathrm{O}_{2} \mathrm{P}$ foram similares.

Alguns trabalhos demonstram em animais eficientes menor produção de calor em relação aos ineficientes, estimada por outras metodologias (Basarab et al., 2003; Almeida, 2005; Nkrumah et al., 2006). A produção de calor foi $21 \%$ menor para animais de baixo CAR, quando comparados 
a animais de alto CAR e, $10 \%$ menor para animais de médio CAR, quando comparados a animais de alto CAR, mostrando que um dos fatores que pode explicar a melhor eficiência dos animais baixo CAR é o menor gasto energético com produção de calor (Nkrumah et al., 2006). Menor produção de calor nos animais eficientes está relacionado com menor exigência de mantença, e isto pode ser consequência de muitos mecanismos biológicos, como baixa produção de metano durante a digestão (Nkrumah et al., 2006), baixa atividade física (Barea et al., 2010; Luiting et al., 1994) e menor resposta ao estresse (Knott et al., 2008, 2010).

\section{CONSIDERAÇÕESFINAIS}

Apesar de existirem poucos estudos relacionados ao tema, os resultados

\section{BIBLIOGRAFIA}

Aharoni, Y.; Brosh, A. and Harari, Y. 2005. Night feeding for high-yielding dairy cows in hot weather: effects on intake, milk yield and energy expenditures. Livest Prod Sci, 92: 207-219.

Aharoni, Y.; Brosh, A. and Ezra, E. 1999. Effects of heat load and photoperiod on milk yield and composition in three dairy herds in Israel. J Anim Sci, 69: 37-47.

Aharoni, Y.; Brosh, A. and Kafchuk, E. 2006. The efficiency of utilization of metabolizable energy for milk production: a comparison of Holstein with F1 Montbeliarde X Holstein cows. J Anim Sci, 82: 101-109.

Aharoni, Y.; Brosh, A.; Kourilov, P. and Arieli, A. 2003. The variability of the ratio of oxygen consumption to heart rate in cattle and sheep at different hours of the day and under different heat load conditions. Livest Prod Sci, 79: 107117.

Ahola, J.K.; Skow, T.A.; Hunt, C.W. and Hill, R.A. 2011. Relationship between residual feed intake and end product palatability in longissumus steaks from steers sired by Angus bulls divergent for intramuscular fat expected progeny difference. Prof Anim Sci. 27: 109-115.

Almeida, R. 2005. Consumo e eficiência alimentar disponíveis na literatura são bastante favoráveis à utilização da mensuração diária de frequência cardíaca combinada com a calibração individual do consumo de oxigênio por batimento, como um método útil para determinar a produção de calor em ruminantes. Dessa forma, esta metodologia poderia ser explorada - para a identificação de animais com menor produção de calor em um rebanho de seleção, ou seja, aqueles com menor exigência de mantença, para dado nível de produção e consumo, consequentemente estes serão mais eficientes quanto à utilização do alimento. Devido à importância fisiológica do gasto energético e da motivação da indústria para produzir animais mais eficientes, é importante, antes de optar por utilizar determinado índice ou método, que as relações com outras características de produção sejam conhecidas.

de bovinos em crescimento. Tese (Doutorado em Ciência Animal e Pastagens). Escola Superior de Agricultura "Luiz de Queiroz". Universidade de São Paulo. Piracicaba. 181 pp.

Andrews, R.B. 1971. Net heart rate as a substitute for respiratory calorimetry. Am J Clin Nutr, 24: 1139-1147.

Archer, J.A.; Arthur, P.F.; Herd, R.M.; Parnell, P.F. and Pitchford, W.S. 1997. Optimum postweaning test for measurement of growth rate, feed intake, and feed efficiency in British breed cattle. J Anim Sci, 75: 2024-2032.

Archer, J.A. and Bergh, L. 2000. Duration of performance tests for growth rate, feed intake and feed efficiency in four biological types of beef cattle. Livest Prod Sci, 65: 47-55.

Archer, J.A.; Richardson, E.C.; Herd, R.M. and Arthur, P. 1999. Potential for selection to improve efficiency of feed use in beef cattle: a review. Aust J Agr Sci, 50: 147-161.

Arieli, A.; Kalouti, A.; Aharoni, Y. and Brosh, A. 2002. A. Assessment of energy expenditure by daily heart rate measurement - validation with energy accretion in sheep. Livest Prod Sci, 78: 99-105.

Arthur, P.F.; Archer, J.A. and Herd, R.M. 2004. 


\section{PULSO DE OXIGÊNIO, PRODUÇÃO DE CALOR E CONSUMO ALIMENTAR RESIDUAL}

Feed intake and efficiency in beef cattle: Overview of recent Australian research and challenges for the future. Aust J Exp Agr, 44: 361-369.

Arthur, P.F.; Archer, J.A.; Johnston, D.J.; Richardson, E.C. and Parnell, P.F.2001b. Genetic and phenotypic variance and covariance components for feed intake, feed efficiency and other postweaning traits in Angus cattle. $J$ Anim Sci, 79: 2805-2811.

Arthur, P.F.; Archer, J.A.; Herd, R.M. and Melville, G.J. 2001a. Response to selection for net feed intake in beef cattle. Association for the Advancement of Animal Breeding and Genetics. Queenstown. Proceedings... 14: 135-138.

Barea, R.; Dubois, S.; Gilbert, H.; Sellier, P.; Van Milgen, J. and Noblet, J. 2010. Energy utilization in pigs selected for high and low residual feed intake. J Anim Sci, 88: 2062-2072.

Basarab, J.A.; Price, M.A.; Aalhus, J.L.; Okine, E.K.; Snelling, W.M. and Lyle, K.L. 2003. Residual feed intake and body composition in young growing cattle. Can J Anim Sci, 83: 189-204.

Berry, D.P. 2008. Improving feed efficiency in cattle with residual feed intake. In: $P$. Garnsworthy (Ed.). Recent advances in Animal Nutrition. University of Nottingham Press. Nottingham, UK. pp. 67-99.

Berry, D.P. and Crowley, J.J. 2013. Genetics of feed efficiency in dairy and beef cattle. J Anim Sci, 91: 1594-1613.

Bishop, M.D.; Davis, M.E.; Harvey, W.R.; Wilson, G.R. and Van Stavern, B.D. 1991. Divergent selection for postweaning feed conversion in Angus beef cattle: II. Genetic and phenotypic correlations and realized heritability estimate. $J$ Anim Sci, 69: 4360-4367.

Bradfield, R.B.; Chan, H.; Bradfield, N.E. and Payne, P.R. 1971. Energy expenditure and heart rates of Cambridge boys at school. Am J Clin Nutr, 24: 1461-1466.

Brosh, A. 2007. Heart rate measurements as an index of energy expenditure and energy balance in ruminants: A review. J Anim Sci, 85: 12131227.

Brosh, A.; Aharoni, Y.; ShargaL, E.; Sharir, B.; Gutman, M. and Choshniak, I. 2004. Energy balance of grazing beef cattle in Mediterranean pasture, the effects of stocking rate and season. 2. Energy expenditure as estimated from heart rate and oxygen consumption, and energy balance. Livest Prod Sci, 90: 101-115.

Brosh, A.; Aharoni, Y.; Degen, A.; Wright, D. and Young, B.A. 1998a. Estimation of energy expenditure from heart rate measurements in cattle maintained under different conditions. J Anim Sci, 76: 3054-3064.

Brosh, A.; Aharoni, Y.; Degen, A.; Wright, D. and Young, B.A. 1998b. Effects of solar radiation, dietary energy, and time of feeding on thermoregulatory responses and energy balance in cattle in a hot environment. J Anim Sci, 76: 2671-2677.

Brosh, A.; Aharoni, Y. and Holzer, Z. 2002. Energy expenditure estimation from heart rate: Validation by long-term energy balance measurement in cows. Livest Prod Sci, 77: 287-299.

Carstens, G.E.; Johnson, D.E.; Johnson, K.A.; Hotovy, S.K. and Szymanski, T.J. 1989. Genetic variation in energy expenditures of monozygous twin beef cattle at 9 and 20 months of age. Energy Metabolism of Farm Animals. $11^{\text {th }}$ Symposium. Luteren. Proceedings. pp. 312315.

Castilhos, A.M.; Branco, R.H.; Razook, A.G.; Bonilha, S.F.M.; Mercadante, M.E.Z. and Figueiredo, L.A. 2011. Test post-weaning duration for performance, feed intake and feed efficiency in Nellore cattle. Rev Bras Zootecn, 40: 301-307.

Castro Bulle, F.C.; Paulino, F.C.P.; Sanches, A.C. and Sainz, R.D. 2007. Growth, carcass quality, and protein and energy metabolism in beef cattle with different growth potentials and residual feed intakes. J Anim Sci, 85: 928-936.

Ceesay, S.M.; Prentice, A.M.; Day, K.C.; Murgatroyd, P.R.; Goldberg, G.R.; Scott, W. and Spurr, G.B. 1989. The use of heart rate monitoring in the estimation of energy expenditure: a validation study. Brit J Nutr, 61: 175-186.

Chaves, A.S.; Nascimento, M.L.; Tullio, R.R.; Alencar, M.M de; Rosa, A.N. and Lanna, D.P.D. 2013. Relationship of residual feed intake with heart rate and heat production in Nellore steers. Joint Annual Meeting of Animal Science Society. Indianapolis. J Anim Sci, 91: 18-19.

Chaves, A.S.; Feltrin, G.B.; Nascimento, M.L.; Tullio, R.R.; Alencar, M.M de; Rosa, A.N. and Lanna, D.P.D. 2014. Relationship of feed efficiency indexes, oxygen consumption, heart rate and blood parameters in Nellore steers. 51 


\section{CHAVES, FELTRIN, NASCIMENTO E LANNA}

Reunião Anual da Sociedade Brasileira de Zootecnia. Aracaju.

Close, W.H. 1990. The evaluation of feedstuffs through calorimetry studies. In: Wiseman, J.; Cole, D.J.A. (Eds.). Feedstuffs Evaluation. Butterworths. London. pp. 21-39.

Crowley, J.J.; McGee, M.; Kenny, D.A.; Crews, D.H.; Evans Jr., R.D. and Berry, D.P. 2010. Phenotypic and genetic parameters for different measures of feed efficiency in different breeds of Irish performance-tested beef bulls. J Anim Sci, 88: 885-894.

Del Claro, A.C. 2011. Avaliação do consumo alimentar residual de bovinos Nelore dentro e entre grupos contemporâneos. Dissertação (Mestrado em Produção Animal Sustentável). Instituto de Zootecnia. APTA/SAA. Nova Odessa-SP. 68 pp.

Fancy, S.G.; Blanchard, J.M.; Holleman, D.F.; Kokjer, K.J. and White, R.G.1986. Validation of doubly labeled water method using a ruminant. Am J Physiol, 251: 143-149.

Ferrannini, E. 1988. The theoretical basis of indirect calorimetry. A review. Metabolism, 37: 287301.

Ferrell, C.L. and Jenkins, T.G. 1984. Energy utilization by mature, nonpregnant, nonlactating cows of different types. J Anim Sci, 58: 234-243.

Hafla, A.N.; Cartens, G.E.; Forbes, T.D.A.; Tedeschi, L.O.; Bailey, J.C.; Walter, J.T. and Johnson, J.R. 2013. Relationships between postweaning residual feed intake in heifers and forage use, body composition, feeding behavior, physical activity, and heart rate of pregnant beef females. J Anim Sci, 91: 5353-5365.

Herd, R. M.; Oddy, V. H. and Richardson, C. 2004. Biological basis for variation in residual feed intake in beef cattle. 1. Review of potential mechanisms. Aust J Exp Agr, 44: 423-430.

Herd, R.M.; Archer, J.A. and Arthur, P.F. 2003. Reducing the cost of beef production through genetic improvement in residual feed intake: Opportunity and challenges to application. J Anim Sci, 81: 9-17.

Herd, R.M. and Bishop, S.C. 2000. Genetic variation in residual feed intake and its association with other production traits in British Hereford cattle. Livest Prod Sci, 63: 111-119.

Hill, R.A. and Herd, R.M. 2001. Variation in the endocrine system that might influence feed efficiency. In: Feed efficiency in beef cattle. Feed Efficiency Workshop. University of New England. Armidade, NSW. Proceedings. pp. 5154.

Hotovy, S.K.; Johnson, K.A.; Johnson, D.E.; Carstens, G.E.; Bourdon, R.M. and Siedel, G.E. 1991. Variation among twin beef cattle in maintenance energy requirements. J Anim Sci, 69: 940-946.

Jensen, J.; Mao, I.L.; Andersen, B.B. and Madsen, P. 1992. Phenotypic and genetic relationships between residual energy intake and growth, feed intake, and carcass traits of young bulls. J Anim Sci, 70: 386-395.

Kleiber, M. 1961. The fire of life. An introduction to animal energetics. Wiley \& Sons. NY. 454 pp.

Knott, S.A.; Cumminsb, L.J.; Dunshea, F.R. and Leury, B.J. 2008. Rams with poor feed efficiency are highly responsive to an exogenous adrenocorticotropin hormone (ACTH) challenge. Domest Anim Endocrinol, 34: 261-268.

Knott, S.A.; Cummins, L.J.; Dunshea, F.R. and Leury, B.J. 2010. Feed efficiency and body composition are related to cortisol response to adrenocorticotropin hormone and insulininduced hypoglycemia in rams. Domest Anim Endocrinol, 39: 137-146.

Koch, R.M.; Swiger, L.A.; Chambers, D. and Gregory, K.E. 1963. Efficiency of feed use in beef cattle. J Anim Sci, 22: 486-494.

Lancaster, P.A.; Carstens, G.E.; Ribeiro, F.R.B.; Tedeschi, L.O. and Crews, D.H. 2009. Characterization of feed efficiency traits and relationships with feeding behavior and ultrasound carcass characteristics in growing bulls. J Anim Sci, 87: 1528-1539.

Livingstone, M.B.E. 1997. Heart-rate monitoring: The answer for assessing energy expenditure and physical activity in population studies. Brit J Nutr, 78: 869-871.

Luiting, P.; Urff, E.M. and Vestergen, M.W.A. 1994. Between animal variations in biological efficiency as related to residual feed consumption. Neth $\mathrm{J}$ Agr Sci, 42: 59-67.

McCrory, M.A.; Mole, P.A.; Nommsen-Rivers, L.A. and Dewey, K.G. 1997. Between-day and withinday variability in the relation between heart rate and oxygen consumption: effect on estimation of energy expenditure by heart-rate monitoring. Am J Clin Nutr, 66: 18-25. 


\section{PULSO DE OXIGÊNIO, PRODUÇÃO DE CALOR E CONSUMO ALIMENTAR RESIDUAL}

Nagy, K.A. 1989. Field bioenergetics: Accuracy of models and methods. Phys Zool, 62: 237-251.

NRC. National Research Council. 1984. Nutrient requirements of beef cattle. $6^{\text {th }}$ ed. National Academic Press. Washington. 90 pp.

NRC. National Research Council. 1996. Nutrient requirements of beef cattle. $7^{\text {th }}$ ed. National Academic Press. Washington. 244 pp.

Nkrumah, J.D.; Okine, E.K.; Mathison, G. W.; Schmid, K.; LI, C.; Basarab, J.A.; Price, M.A.; Wang, Z. and Moore, S.S. 2006. Relationships of feedlot feed efficiency, performance, and feeding behaviour with metabolic rate, methane production, and energy partitioning in beef cattle. J Anim Sci, 84: 145-153.

Nkrumah, J.D.; Basarab, J.A.; Wang, Z.; Li, C.; Price, M.A.; Okine, E.K.; Crews, D.H. and Moore, S.S. 2007. Genetic and phenotypic relationships of feed intake and different measures of feed efficiency with growth and carcass merit of beef cattle. J Anim Sci, 85: 2711-2720.

Paddock, Z.D. 2010. Energy expenditure in growing heifers with divergent residual feed intake phenotypes. effects and interactions of metaphylactic treatment and temperament on receiving steers. Thesis (Master of Science). Texas A\&M University. College Station. Texas, EUA. 62 pp.

Payne, P.R.; Wheeler, E.F. and Salvosa, C.B. 1971. Prediction of daily energy expenditure from average pulse rate. Am J Clin Nutr, 24: 161-170.

Retallick, K.M.; Faulkner, D.B.; Rodriguez-Zas, S.L.; Nkrumah, J.D. and Shike, D.W. 2013. Relationship among performance, carcass, and feed efficiency characteristics, and their ability to predict economic value in the feedlot. J Anim Sci, 91: 5954-5961.

Robinson, D.L. and Oddy, V.H. 2004. Genetic parameters for feed efficiency, fatness, muscle area and feeding behaviour of feed lot finished beef cattle. Livest Prod Sci, 90: 255-270.

Rodriguez, N.M.; Campo, W.E.; Lachica, M.L.; Borge, I.; Gonçalve, L.C.; Borges, A.L.C.C. and Saliba, E.O.S. 2007. A calorimetry system for metabolism trials. Arq Bras Med Vet Zoo, 59: 495-500.

Schenkel, F.S.; Miller, S.P. and Wilton, J.W. 2004. Genetic parameters and breed differences for feed efficiency, growth, and body composition traits of young beef bulls. Can J Anim Sci, 84: 177-185

Swanson, K. and Miler, S. 2008. Factors regulating feed efficiency and nutrient utilization in beef cattle. In: France, J.; Kebreab, E. Mathematical Modelling Animal Nutrition. CAB International. Cambridge. pp. 419-441.

Taylor, C. R.; Heglund, N.C. and Maloiy, G.M.O. 1982. Energetics and mechanics of terrestrial locomotion as a function of speed and body size in birds and mammals. J Exp Biol, 97: 1-21.

Thompson, W.R.; Meiske, J.C.; Goodrich, R.D.; Rust, J.R. and Byers, F.M. 1983. Influence of body composition on energy requirements of beef cows during winter. J Anim Sci, 56: 12411252.

Van Soest, P.J. 1982. Nutritional ecology of the ruminant. $\mathrm{O} \&$ Books. Corvalis.

Wang, Z.; Nkrumah, J.D.; Li, C.; Basarab, J.A.; Goonewardene, L.A.; Okine, E.K.; Crews, D.H. and Moore, S.S. 2006. Test duration for growth, feed intake, and feed efficiency in beef cattle using the GrowSafe system. J Anim Sci, 84: 2289-2298.

Warnold, L. and Lenner, R.A. 1977. Evaluation of the heart rate method to determine the daily energy expenditure in disease. A study in juvenile diabetics. Am J Clin Nutr, 30: 304-315.

Webster, A.J. 1967. Continuous measurement of heart rate as an indicator of the energy expenditure of sheep. Brit J Nutr, 21: 769-785.

Yamamoto, S. 1989. Estimation of heat production from heart rate measurement of free living farm animals. Ibaraki, 23:134-143, Disponível em: <http://journalseek.net/cgi-bin/journalseek/ journalsearch.cgi? field=issn\&query $=0021$ 3551> Acesso em: 17 fevereiro 2013.

Yamamoto, S.; McLean, J.A. and Downie, A.J. 1979. Estimation of heat production from heart rate measurements in cattle. Brit J Nutr, 42: 507-513.

Young, B.A. and Corbett, J.L. 1972. Maintenance energy of grazing sheep in relation to herbage availability. Aust J Agr Res, 23: 57-76.

Young, B.A. and Webster, M.E.D. 1963. A technique for the estimation of energy expenditure in sheep. Aust J Agr Res, 14: 867-873. 